\title{
Compressibility Modified RANS Simulations for Noise Prediction of Jet Exhausts with Chevron
}

\author{
Y. Jin ${ }^{1,2}, X . \operatorname{Han}^{1,2 \dagger}$ and P. Fan $^{1}$ \\ ${ }^{1}$ College of Energy and Power Engineering, Nanjing University of Aeronautics and Astronautics, Nanjing \\ 210016, China \\ ${ }^{2}$ Aero-engine Thermal Environment and Structure Key Laboratory of Ministry of Industry and Information \\ Technology, China
}

†Corresponding Author Email: xshan@nuaa.edu.cn

(Received June 16, 2020; accepted November 4, 2020)

\begin{abstract}
The impact of compressibility modified RANS turbulence closures is investigated for high subsonic round and chevron jet flows with Mach $=0.9$ and $\mathrm{Re}=1.03 \times 10^{6}$, including the predicted acoustic noise generation. The well-documented chevron jet flow and noise cases, namely NASA SMC000 and SMC006 are selected as the simulation configurations. Two compressibility RANS closures are considered, which are based on the k- $\varepsilon$ turbulence model. The first type only considers the compressibility dissipation rate, and the second type accounts for three modifications of compressibility dissipation rate, pressure dilation and production limiter. The acoustic noise is calculated employing the SNGR (Stochastic Noise Generation and Radiation) method using the flow prediction of the three-dimensional RANS simulations. The results show that both of the two types of compressibility modified RANS models improve the accuracy of the mean flow and turbulence quantities. This results in more accurate jet noise predictions than with the standard RANS model. The first type modification is found to be moderate and the second type is remarkable. The noise results by the second type model, i.e. Sarkar2 model, agree with the experimental data quite well. For the mean flow field, the compressibility modified model (Sarkar2 model) estimates a shorter potential jet core, and improved predictions of the velocity in the downstream region are observed. The study demonstrates the importance of considering the compressibility modified RANS closure for the noise prediction of high-speed jets via the comparison to experimental data. Hence, the SNGR method is found to be cost effective for jet noise prediction, when compared to other approaches.
\end{abstract}

Keywords: Compressibility modification; Chevron nozzle jet; Noise reduction; Aeroacoustics; RANS simulation.

\section{NOMENCLATURE}

$\begin{array}{ll}C_{\varepsilon l} & \text { model constant } \\ C_{\varepsilon 2} & \text { model constant } \\ C_{\mu} & \text { model constant } \\ D & \text { diameter at the nozzle exit } \\ k & \text { turbulent kinetic energy } \\ \mathrm{Ma} & \text { Mach number } \\ M_{c} & \text { convective Mach number } \\ M_{t} & \text { turbulent Mach number } \\ p & \text { pressure } \\ P_{k} & \text { turbulence production term }\end{array}$

\section{INTRODUCTION}

With the development of aviation industry and high demand of environmental protection, the jet noise

$\begin{array}{ll}\operatorname{Re} & \text { Reynolds number } \\ S & \text { strain rate } \\ U & \text { axial velocity } \\ & \\ \varepsilon & \text { dissipation rate of turbulent kinetic energy } \\ \mu & \text { molecular viscosity } \\ \mu_{t} & \text { turbulent viscosity } \\ \rho & \text { density } \\ \sigma_{k} & \text { model constant } \\ \sigma_{\varepsilon} & \text { model constant }\end{array}$

of aero-engine has become one of the major concerns in the aircraft manufacturing industry (Tam et al. 2008). A large number of experimental and numerical studies have been carried out to 
understand the mechanisms of aero-acoustic noise generation (Tam et al. 2008; Freund, 2001; Bahman-Jahromi et al. 2019; Zuo et al. 2019) and its reduction (Henderson 2010; Maizi et al. 2017; Zhao et al. 2018; Semlitsch et al. 2019). For the simplicity and the effectivity for noise reduction, chevron nozzles (Bastos et al. 2017; Xia et al. 2009) are one of the most popular noise reduction strategies. They have been successfully applied by the aviation industry.

From the view point of numerical studies, jet noise prediction is one of the fundamental problems for both academia and industrial applications. The classical acoustic analogy was firstly proposed by Lighthill (Lighthill 1954), which provides the theoretical foundation for the study of jet noise. Balsa et al. (1978) proposed a methodology combining the turbulence quantities from the RANS (Reynolds-Averaged Navier-Stokes) calculations and the jet noise characteristics to study the jet noise generation. Tam and Auriault (1999) suggested a semi-empirical formula for the noise prediction of small-scale jets. This method was applied to round jets at a high subsonic condition (Rosa et al. 2013; Raizada and Morris 2006) with the RANS turbulence models. It was found that turbulence models have a significant impact on the noise prediction and that the $k-\varepsilon$ turbulence model works well for jet flow and noise prediction. Various jet noise methods (Engel et al. 2014; Venkatesh and Self 2015) have been proposed since the 1990's based on RANS calculations associated with turbulence length scales and time scales. Later, a novel approach to predict the jet noise was proposed using the linearized Euler equations as an acoustic analogy together with the source terms reconstructed from the turbulent fields of RANS calculations. One such method is the Stochastic Noise Generation and Radiation (SNGR) method developed by Bailly et al. (1995; 1997). It was applied to study serials of subsonic jet noise and good predictions were obtained.

In recent years, with the significant development of the computational capability, high-fidelity turbulence methods are becoming applicable for jet noise predictions, such as LES (Large Eddy Simulation), hybrid RANS/LES methods. It has been reviewed in recent literature (Bres and Lele 2019). Xia et al. (2009) applied LES to study the jet noise with 0.9 Mach number using around 12.5 million computational grids. Uzun and Hussaini (2012) applied LES method to study the jet noise, where the mesh size is as high as 370 million. Although LES has significantly improved in the past two decades and provided a better understanding of jet noise, LES calculations require forbiddingly amounts of computational resources to allow geometrical optimization in engineering applications, such as presented by Semlitsch et al. (2018). Hence, LES remains too costly for the evaluation of large scenarios reducing jet noise. In contrary, the RANS method is suitable for jet noise predictions of chevron nozzles with high Reynolds number.

All RANS-based jet noise methods rely on the turbulent flow field prediction, which are significantly affected by the particular RANS turbulence closure. For turbulent flow, with increasing Mach number, the compressibility effects become more and more significant (Gatski and Bonnet 2009; Chassaing et al. 2002; Krishnamurty and Shyy 1997).

Early studies about compressible turbulence are mainly based on theoretical analysis (Kovasznay 1953) and experimental observation (Demetriades 1970). Later, extensive experimental measurements are conducted about the compressible shear layers such as jets and mixing layers, and some results are summarized in the references (Lele 1994; Slessor et al. 2000). One important observation is that the spreading rate of the mixing layers reduces significantly with increasing the convective Mach number $\left(M_{c}\right)$. Between $M_{c}=0.5$ and $M_{c}=1.0$, there is a reduction by more than a factor of two (Gatski and Bonnet 2009). Such reduction leads to severe concerns about relevant compressible engineering flows, such as in supersonic flow and combustion. To account the compressibility level, an important parameter, i.e. turbulent Mach number $M_{t}$, is introduced and it is pivotal in the development of both dilatation dissipation and pressure-dilatation terms by different groups (Sarkar et al. 1991; Sarkar 1992; Zeman, 1991; Erlebacher et al. 1990). Those effects of dilatation dissipation and pressuredilatation have been found to affect the flow field significantly, especially for the turbulent mixing process. The Sarkar's model (Sarkar 1992) is found to work well to accurately predict the spreading rate of the compressible mixing layers.

Another important modification for compressible flow simulation is relevant to shock unsteadiness. It is found that the frequency of the fluctuations produced by the shock motion is much lower than the characteristic turbulence frequencies in the incoming boundary layers (Dussauge et al. 2006). Sinha et al. (2003) proposed a model with a shock unsteadiness effect based on the DNS results of isotropic turbulence interacting with a shock. However, the model is strictly applicable only when the mean flow on either side of the shock is uniform. On the basis, Han et al. (2008) proposed a compressibility model including both the shock unsteadiness, dilatation dissipation and pressuredilatation. The model has been successfully applied for complex turbulent combustion simulations (Han et al. 2015).

The studies show that, for the high speed jet, the compressibility should play an important role for the noise. However, for the jet noise prediction based on RANS method, there are a few studies considering the compressibility effect. On the basis, the present study aims to investigate the compressibility effects for jet flow and jet noise predicti on in the RANS framework. To predict the noise characteristics, the SNGR method is applied.

The well-studied chevron jet flow and noise cases, namely the NASA SMC000 and SMC006 are selected as the test configurations. Those have been studied extensively (Bridges and Brown 2004; 
Bridges and Wernet 2011). The jet Mach number is 0.9 and the Reynolds number is around $10^{6}$. There are many numerical studies (Xia et al. 2009; Engel et al. 2014; Uzun and Hussaini 2012) replicating the experimental investigations.

The paper is organized as follows. The physical and mathematical models are presented firstly, followed by the numerical setup and details. The computational result and discussion are described thereafter. Finally, the major findings of the current work are summarized and concluded.

\section{Physical And Mathematical MODELS}

\subsection{Turbulence Models Compressibility Modifications}

with

It is known that the $k-\varepsilon$ turbulence model is suitable for jet flow simulation (Mihaescu et al. 2012). The standard $k-\varepsilon$ model has the forms as:

$$
\begin{aligned}
& \frac{D(\bar{\rho} k)}{D t}=P_{k}-\bar{\rho} \varepsilon+\frac{\partial}{\partial x_{j}}\left[\left(\mu+\frac{\mu_{t}}{\sigma_{k}}\right) \frac{\partial k}{\partial x_{j}}\right] \\
& \frac{D(\bar{\rho} \varepsilon)}{D t}=\frac{\varepsilon}{k}\left(C_{\varepsilon 1} P_{k}-C_{\varepsilon 2} \bar{\rho} \varepsilon\right)+\frac{\partial}{\partial x_{j}}\left[\left(\mu+\frac{\mu_{t}}{\sigma_{\varepsilon}}\right) \frac{\partial \varepsilon}{\partial x_{j}}\right] \\
& -\overline{\rho u_{i}^{\prime \prime \prime} u_{j}^{\prime \prime}}=\mu_{t}\left(\frac{\partial \tilde{u}_{i}}{\partial x_{j}}+\frac{\partial \tilde{u}_{j}}{\partial x_{i}}\right)-\frac{2}{3}\left(\bar{\rho} k+\mu_{t} \frac{\partial \tilde{u}_{m}}{\partial x_{m}}\right) \delta_{i j} \\
& \mu_{t}=C_{\mu} \bar{\rho} \frac{k^{2}}{\varepsilon}
\end{aligned}
$$

where ' $\sim$ ' denotes the Favre average, ' "' for Favre fluctuations, '-' for Reynolds average and ' ', ' for Reynolds fluctuations. The default values of model constants are $C_{\varepsilon 1}=1.44, C_{\varepsilon 2}=1.92, C_{\mu}=0.09, \sigma_{k}=$ $1.0, \sigma_{\varepsilon}=1.3$.

It should be noted that Eqs. (1) - (2) are derived with incompressible flow assumptions, which means that for high-speed compressible flow, the equations should be reformulated. Considering the flow compressibility, the governing equation of turbulent kinetic energy, $k$, can be rewritten as (Chassaing et al. 2002; Krishnamurty and Shyy 1997):

$\frac{D(\bar{\rho} k)}{D t}=P_{k}-\bar{\rho} \varepsilon+\frac{\partial}{\partial x_{j}}\left[\left(\mu+\frac{\mu_{t}}{\sigma_{K}}\right) \frac{\partial k}{\partial x_{j}}\right]+\overline{p^{\prime} \frac{\partial u_{i}^{\prime \prime}}{\partial x_{i}}}$

An additional term appears in Eq. (5), which is the so-called pressure-dilatation term. It is one of the important compressibility effects.

For compressible flow, previous studies (Chassaing et al. 2002; Krishnamurty and Shyy 1997) show that the dissipation rate, $\varepsilon$, can be expressed as the sum of the non-divergence part, $\varepsilon_{s}$, and the part with non-zero divergence, $\varepsilon d$, i.e.:

$\bar{\rho} \varepsilon=\bar{\rho} \varepsilon_{s}+\bar{\rho} \varepsilon_{d}$

where $\varepsilon_{d}$ is an important part for the compressibility effects.
It is found that the production term of turbulent kinetic energy in Eqs. (1)-(2) is generally overestimated for compressible flows, especially in the proximity of shocks (Han et al. 2008). To partly reduce the consequence, a limiter of the production term was introduced, i.e.:

$$
P_{k}=\min \left(P_{k}^{\text {org }}, P_{k}^{\text {limiter }}\right)
$$

where 'org' means the original formula to calculate the turbulent kinetic energy production, and 'limiter' is a modeled limiter to limit the production term.

In the present study, the Sarkar's models (Sarkar et al. 1991; Sarkar 1992) are applied to estimate the compressibility dissipation rate and the pressuredilatation term. The terms have the forms:

$\varepsilon_{d}=\alpha_{1} \varepsilon_{s} M_{t}^{2}$

$\overline{p^{\prime} \frac{\partial u_{i}^{\prime \prime}}{\partial x_{i}}}=-\alpha_{3} P_{K} M_{t}^{2}+\alpha_{4} \bar{\rho} \varepsilon_{s} M_{t}^{2}$

where the parameter, $M_{t}$, is the turbulent Mach number, defined as $M_{t}=\sqrt{2 k} / a$. It is an important parameter to describe compressibility effects.

The production limiter is modeled as in our previous work (Han et al. 2008). The production term limiter can be written as:

$P_{k}^{\text {limiter }}=\sqrt{\frac{8}{3}} \bar{\rho} k|S|-\frac{2}{3} \bar{\rho} k \frac{\partial \tilde{u}_{i}}{\partial x_{i}}$

It should be noted that the compressibility dissipation rate and pressure-dilatation are two important compressibility effects. Some of the previous studies consider only the compressibility dissipation rate, i.e. Eq. (8), in the simulations. In the present study, this is the first modification model, called "Sarkarl" model. The second proposed method considers the models in Eqs. (8)(10) which means that the compressibility dissipation, the pressure dilation and the production limiter are all considered. This is the modification model 2, named "Sarkar2" in the following sections.

\subsection{Jet Noise Prediction with SNGR Method}

In the present study, the jet noise is predicted based on RANS calculations. Two noise models are applied as shown in Fig. 1. The first is among the broadband noise source models using Lilley's formula (Lilley 1993). It was developed based on Proudman's formula (Proudman 1952) by accounting for the retarded time difference. The acoustic power due to the unit volume of isotropic turbulence can be estimated as:

$P_{A}=\alpha_{\varepsilon} \rho_{0} \varepsilon M_{t}^{5}$

where $\alpha_{\varepsilon}$ the model constant.

The second approach is based on the SNGR method. Based on the mean turbulent flow field, the turbulence fluctuations can be evaluated by the 
SNGR method. After the turbulence fluctuations are obtained, the near-field acoustic source term can be determined from the synthetic turbulence. Then the far-filed noise can be predicted using the classical Lighthill's acoustic analogy.

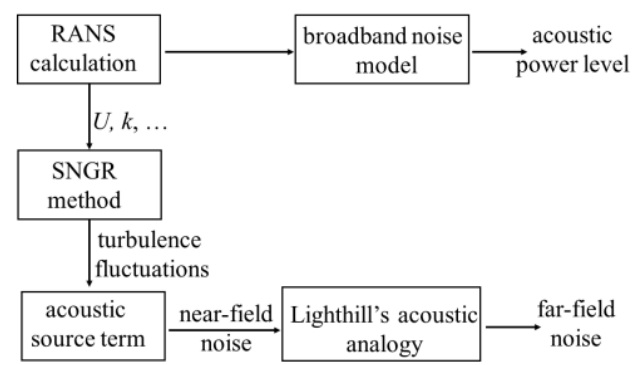

Fig. 1. Jet noise prediction process.

In the SNGR method, turbulence is represented by a technique proposed in previous studies (Bailly et al. 1995; Bailly et al. 1997). The turbulence fluctuations are generated by the sum of $\mathrm{N}$ Fourier modes, i.e.:

$U^{\prime}(x, t)=2 \sum_{n=1}^{N} u_{n} \cos \left[\lambda_{n}\left(x-t U_{c}\right)+\varphi_{n}+\omega_{n} t\right] \sigma_{n}$

where $u_{n}$ is the $n$-th mode amplitude, $\lambda_{n}$ is the $n$-th wavenumber, $U_{c}$ is the local convection velocity, $\varphi_{n}$ is the $n$-th mode phase.

The angular velocity, $\omega_{n}$, is a random vector, which has a distribution given by a Gaussian probability density function. To get the amplitude, the turbulence spectrum is used. Here, the improved von-Karman spectrum is applied, with the form:

$E(\lambda)=A \frac{2 k}{3 \lambda_{e}} \frac{\left(\lambda / \lambda_{e}\right)^{4}}{\left[1+\left(\lambda / \lambda_{e}\right)^{2}\right]^{17 / 6}} \exp \left(-2 \frac{\lambda}{\lambda_{\eta}}\right)$

The turbulence spectrum, $E(\lambda)$, is discretized using the exponential distribution, i.e.:

$d=\frac{\ln \left(\lambda_{N}\right)-\ln \left(\lambda_{1}\right)}{N-1}$

$\lambda_{n}=\exp \left[\ln \left(\lambda_{1}\right)+(n-1) \cdot d\right], n=1,2, \cdots, N$

Based on the previous study (Mesbah et al. 2004) regarding the model parameters, the values, i.e. $N=$ $30, \lambda_{l}=0.2 \lambda_{e, \min }, \lambda_{N}=2 \pi /(6 \Delta x)$, are used in the present study.

\subsection{Acoustic Noise Parameters}

The acoustic power level can be calculated as:

$$
L_{P}=10 \log \left(\frac{P_{A}}{P_{r e f}}\right)
$$

where the reference value is $P_{r e f}=1 \times 10^{-12} \mathrm{~W} / \mathrm{m}^{3}$.

The SPL (Sound Pressure Level) is defined as:

$$
S P L=20 \log \frac{p^{\prime}}{p_{\text {ref }}}
$$

where the reference fluctuation pressure is $p_{\text {ref }}=$ $2 \times 10^{-5} \mathrm{~Pa}$. The sound pressure signal, i.e. SPL, has a frequency spectrum. If the SPL spectrum is integrated over a frequency range, the levels are known as the Overall Sound Pressure Level, i.e. OASPL. The OASPL will be used to evaluate the jet noise.

\section{NUMERICAL SETUP AND DETAILS}

\subsection{RANS Calculation}

The chevron nozzles considered in the present study are the SMC000 and SMC006 cases of the experimental study (Bridges and Brown 2004; Bridges and Wernet 2011). The geometries are shown in Fig. 2. The main geometrical details of the two nozzles are given in Table 1.
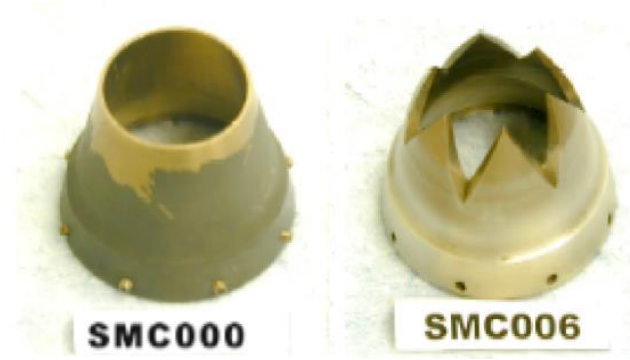

(a)

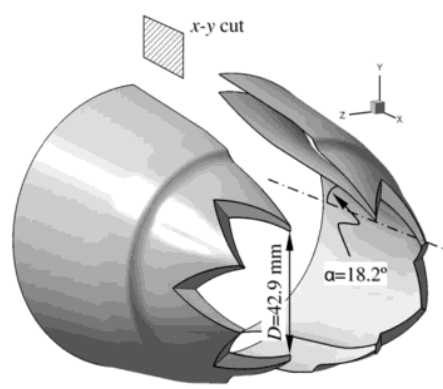

(b)

Fig. 2. Pictures of the investigated jet nozzles (Bridges and Brown 2004; Bridges and Wernet 2011) (a) and the schematic diagram of the chevron nozzle (Xia et al. 2009).

For the present three-dimensional turbulent flow simulations, structured grids are employed as shown in Fig. 3(a) with the coordinate system. The length of computational domain is around $40 \mathrm{D}$, and the outer radius is $15 \mathrm{D}$. The nozzle exit is located $1 \mathrm{D}$ from the inlet boundary and the outlet boundary is located 39D from the nozzle exit. In the near wall region of the nozzle, the non-dimensional distance of $y^{+}$is smaller than 1.0 in order to well resolve the near-wall flow. In the downstream region, the growth ratio of the mesh is 1.15 . The mesh contains about 1.8 million cells for SMC000 case and 2.3 million cells for SMC006 case, respectively, after several simulation tests. One example is shown in Fig. 3(b) of the mesh sensitivity study for the SMC000 case with three different meshes, containing about 1.0 million, 1.8 million and 3.0 million cells. The mesh M2 is chosen for the final 
Y. Jin et al. / JAFM, Vol. 14, No. 3, pp. 793-804, 2021.

Table 1 Geometric detail of the two nozzles

\begin{tabular}{|c|c|c|c|c|}
\hline Nozzle type & $\begin{array}{c}\text { Chevron } \\
\text { Number }\end{array}$ & $\begin{array}{c}\text { Chevron length } \\
(\mathrm{mm})\end{array}$ & $\begin{array}{c}\text { Penetration } \\
\text { angle }\left(^{\circ}\right)\end{array}$ & $\begin{array}{c}\text { Nozzle exit diameter } \\
D(\mathrm{~mm})\end{array}$ \\
\hline SMC000 & 0 & - & - & 50.8 \\
\hline SMC006 & 6 & 22.6 & 18.2 & 47.7 \\
\hline
\end{tabular}

simulations. The mesh is refined in the near wall regions of the nozzle and also in the jet mixing layer.

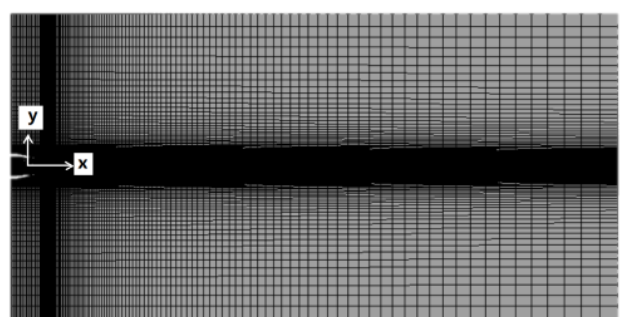

(a)

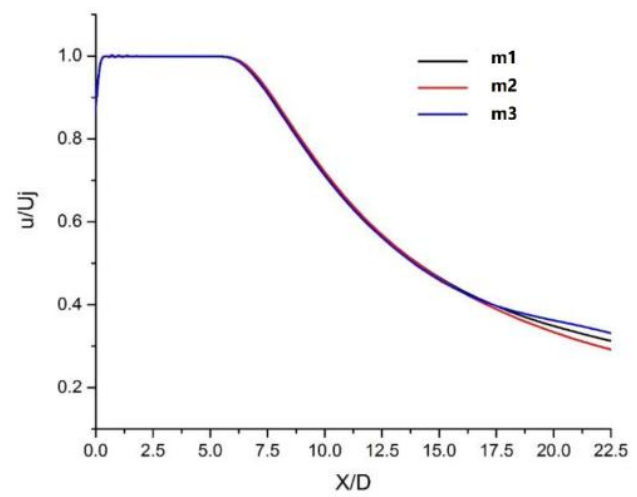

(b)

Fig. 3. Computational mesh (a) in the RANS calculations and the mesh sensitivity study (b) for the SMCO00 case.

Both nozzle configurations are simulated with the same inflow boundary conditions. The total pressure is $178.2 \mathrm{kPa}$, total temperature $288.2 \mathrm{~K}$, static pressure $97.7 \mathrm{kPa}$ and static temperature 280.2 K. Non-slip wall conditions are applied at all the walls. At the exit and the lateral boundary, pressure outlet conditions are applied, i.e. the flow parameters are extrapolated from the internal flow field.

The flow filed is calculated with a general purpose CFD software, ANSYS Fluent (2010). The densitybased solver is applied which solves the governing equations of continuity, momentum, and energy simultaneously as a set, or vector, of equations.

At the solid wall boundary, Enhanced Wall Treatment (EWT) in the CFD code is applied which is a near-wall modeling method that combines a two-layer turbulence model with the enhanced wall functions. In the present study, as the near-wall mesh is fine enough to resolve the viscous sublayer, the enhanced wall treatment is identical to the traditional two-layer zonal turbulence model. In the viscosity-affected near-wall region (i.e. turbulent
Reynolds number smaller than 200), the oneequation low-Re turbulence model of Wolfstein (1969) is employed. In the fully turbulent region (i.e. turbulent Reynolds number larger than 200), the traditional k- $\varepsilon$ turbulence model is applied. More details about the wall treatment in ANSYS Fluent solver can be found in the reference (ANSYS Fluent, 2010) and are not reproduced here for brevity.

\subsection{Acoustic Noise Prediction}

In the noise prediction based on the SNGR method, another computational mesh (see Fig. 4) is applied, which contains about 2.6 million cells after several simulation tests. The acoustic equation is spatially discretized using the high-order finite element method, and the fourth-order Runge-Kutta method is applied for temporal discretization. Based on the near-field acoustic calculations via the SNGR method, the far-field acoustics are determined using Lighthill's acoustic analogy.

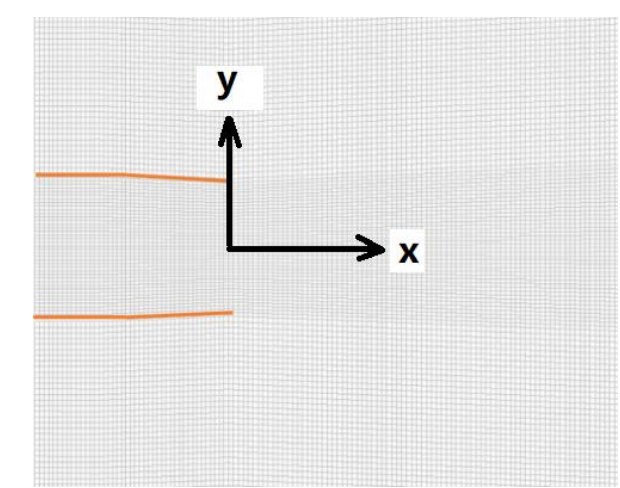

(a)

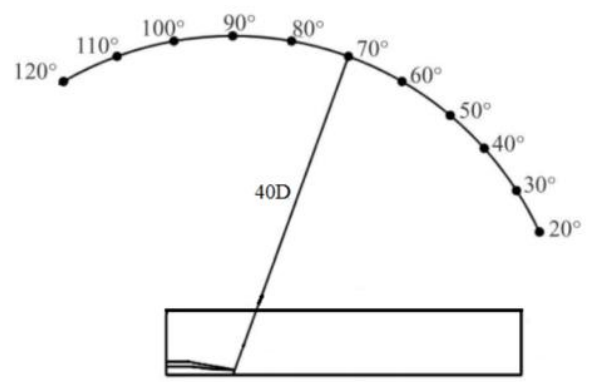

(b)

Fig. 4. Computational mesh in the noise prediction (a) and the locations of the far-field observation points with $D$ the nozzle exit diameter (b).

Computational results of the noise spectra levels and noise directivity are obtained and compared to 
the experimental data. The observer points are located at a distance of 40D in the far-field at the angles varying from $20^{\circ}$ to $120^{\circ}$, as illustrated in Fig. 4.

The noise is calculated with a general purpose software ACTRAN with SNGR method. The results from steady flow simulation obtained from a RANS CFD code are used for the ACTRAN SNGR to synthesize the noise sources and these sources are then imported into an acoustic computation and are then propagated.

\section{RESUlts AND DiscuSSIONS}

\subsection{Turbulent flow Field Results}

The axial velocity distributions at the center line downstream of the nozzle exits are shown in Fig. 5 for both cases (SMC000 and SMC006), which can describe the length of the jet core and the expansion rate of the downstream velocity. In both cases, the standard k- $\varepsilon$ model (denoted as "ke-std") predicts a longer jet core length, and the axial velocity decay rate in the downstream region is too large. Generally, the present turbulence closure with compressibility modifications can improve the predictions significantly, and the results of the Sakar2 model (i.e. with three compressibility modifications) agree well with the experimental data. The Sarkar1 model with one compressibility modification improves the predictions generally, but less than the Sarkar2 model. For the baseline nozzle case, SMC000, the differences between the three model predictions and experiments of the core lengths are about $4.1 \%, 16.1 \%$, and $1.2 \%$, respectively, for the standard $k-\varepsilon$, Sarkar1 and Sarkar2 model. For the SMC006 nozzle case with chevron structure, the differences are significant. As shown in Fig. 5, the decay rate of the axial velocity along the centerline becomes significantly smaller when the compressibility modification models are included in the simulations.

Figure 6 shows the axial velocity distribution contours and the axial velocity plots for the SMC000 case. If not specifically stated, the contours from the top to the down represent the results from the standard $\mathrm{k}-\varepsilon$ model, the Sarkar1 model and the Sarkar2 model, respectively. The prediction of the Sarkar2 model with three compressibility modification terms is the most accurate. The differences between the predictions and the experimental results are low near the nozzle exit, while the differences become larger in the downstream regions.

For the SMC006 nozzle case, as the existence of the chevron structure, the flow field is changed along the axial direction, in which the axial velocity differences between the sections of the chevron tip and the chevron valley is the most significant. The axial velocity contours and the axial velocity distributions at the chevron tip and valley planes are given in Fig. 7 and 8, respectively. Although the geometry structure of the SMC006 is more complicated than the SMC000, the difference of the three models in axial velocity distribution is insignificant. This is probably because the chevron structures highly accelerate the mixing of the jet with the surrounding air and weakens thereby the differences in the simulations. The results also demonstrate that the results by the Sarkar 2 model are still very close to the experimental data.

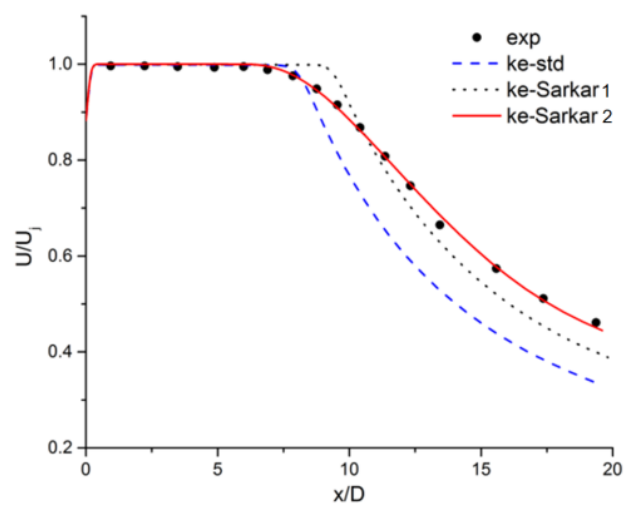

(a)

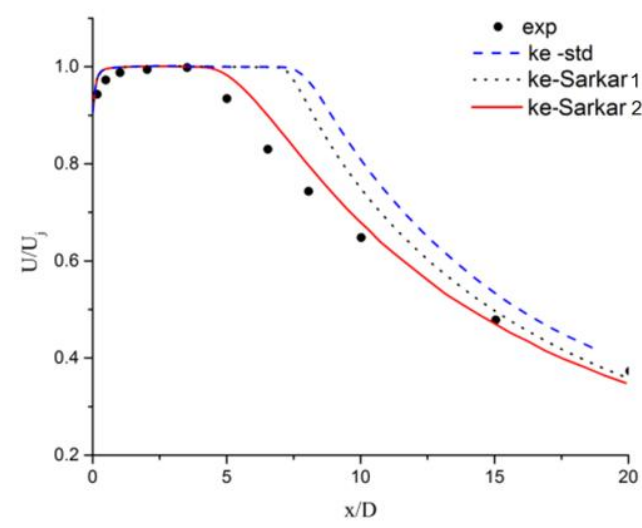

(b)

Fig. 5. Axial velocity distributions at the center line: (a) SMC000 case, and SMC006 case (b).

The experimental data are from the reference by Bridges and Brown (2004).

For the SNGR method, the distribution of the turbulent kinetic energy is very important as it is one of the major noise sources (Bechara et al. 1994; Bose 2013). As the experimental data of the SMC000 case is well documented, the results of the three turbulence models are compared in Fig. 9. It clearly shows that the predictions differ significantly. With the compressibility modifications, the results are significantly improved, and the Sarkar2 model gives the best predictions and also the results agree well with the experiments. The contours show that the turbulent kinetic energy has the largest value inside the shear layers. With the compressibility model, the peak values of the turbulent kinetic energy decrease. At the region very close to the nozzle exit, the turbulent kinetic energy is still over-estimated. This is probably due to the turbulent inlet conditions being not well imposed. However, the tendency of over-estimation is also reported in the previous LES study of the same case (Dhamankar et al. 2016). 


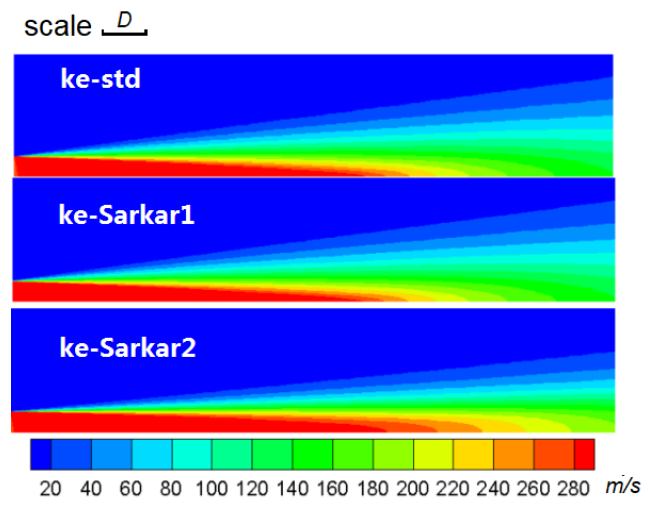

(a)

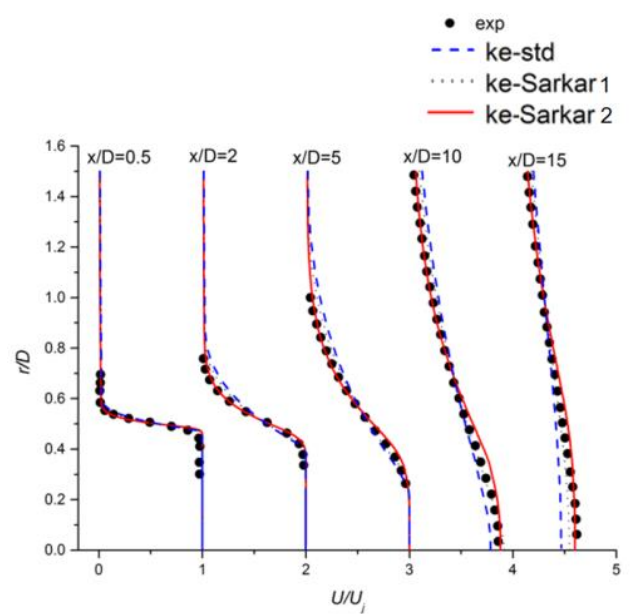

(b)

Fig. 6. Axial velocity distributions for the SMCO00 case. The experimental data are from the reference by Bridges and Brown (2004).

In summary, the prediction accuracy of the standard $\mathrm{k}-\varepsilon$ model for high subsonic jet flows can be significantly improved by the inclusion of compressibility modified models. Considering only the Sarkar1 compressibility correction improves the results insufficiently. The Sarkar2 model with three correction terms is the most accurate for the predictions of the velocity and the turbulent kinetic energy. It can be expected that the flow field by the compressibility modifications can contribute to a more accurate representation of the turbulent kinetic energy synthesized by the SNGR method, which is applied for the acoustic calculations.

\subsection{Acoustic Noise Results}

For the acoustic noise analysis, firstly, the acoustic power levels are explored based on the RANS results using Lilley's formula (Lilley 1993). Figure 10 shows the contours of the acoustic power levels at the central plane for the SMC000 case. Compared with the standard k- $\varepsilon$ model, the Sarkar 1 model and the present Sarkar2 model predict smaller peak values of the acoustic power level, which is also applicable to the SMC006 nozzle case. The acoustic power level contours of the SMC006 case at the chevron tip and valley planes are shown in Fig. 11. Similar to the flow calculations, the differences between the three turbulence models in calculating the acoustic power level of the SMC006 case are smaller than for the SMC000 case. Comparing the two nozzles, it is found that the acoustic power level in the SMC006 case is lower than in the SMC000 case in most of the flow regions.

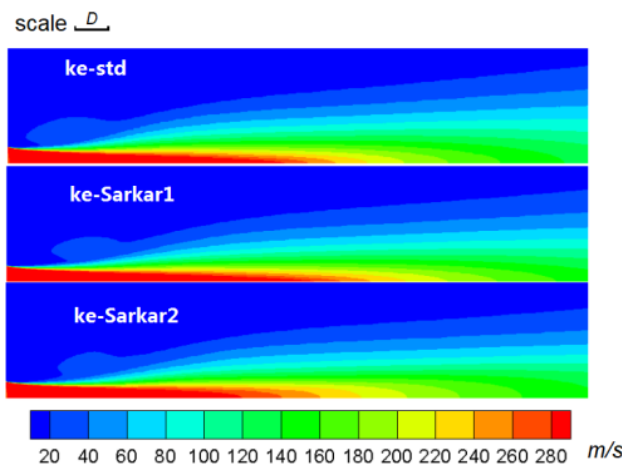

(a)

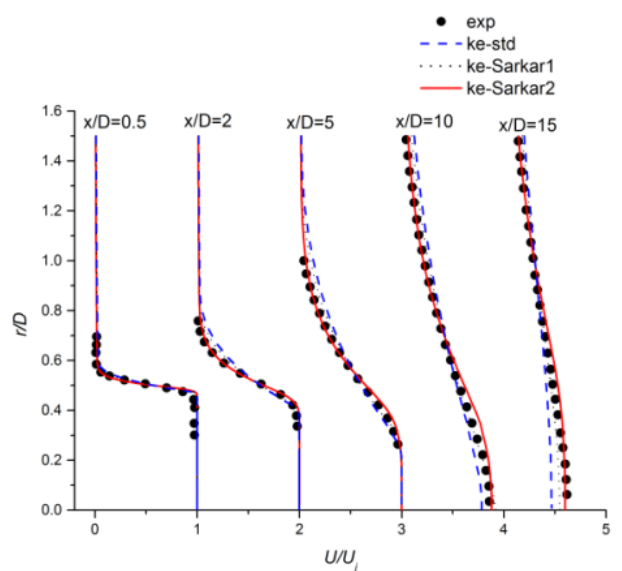

(b)

Fig. 7. Axial velocity distributions for the SMC006 case at the chevron tip plane. The experimental data are from the reference by Bridges and Brown (2004).

The method based on Lighthill's acoustic analogy with the SNGR method is used to calculate the farfield sound pressure levels. Compared to the LES method combined with FW-H approach, the present method can significantly reduce computational costs.

Figure 12 shows the comparisons of the numerical predictions and the experimental data of the farfield total sound pressure level for the SMCO00 nozzle case. It can be seen that the total sound pressure level is gradually reduced with increasing the azimuth angle. The amplitudes at the $20^{\circ}$ azimuth are about $12 \mathrm{~dB}$ higher than that at the $120^{\circ}$ azimuth. All the three turbulence models can well reproduce this trend. However, the standard k- $\varepsilon$ model predicts higher values compared to the experimental observations, where the maximum error is about $6.1 \mathrm{~dB}$ at the location of $70^{\circ}$ azimuth. The noise predictions of the two modified closure models are better than those of the standard k- $\varepsilon$ model. The differences to the experimental data are 
small and thereby, consistent with the observations from the broadband noise model analysis. The Sarkar1 model can improve the predictions while the present Sarkar2 model is the most accurate compared to the experimental data.

scale $D$

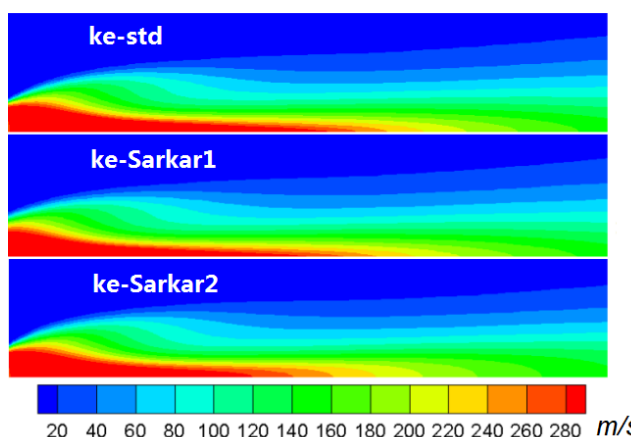

(a)

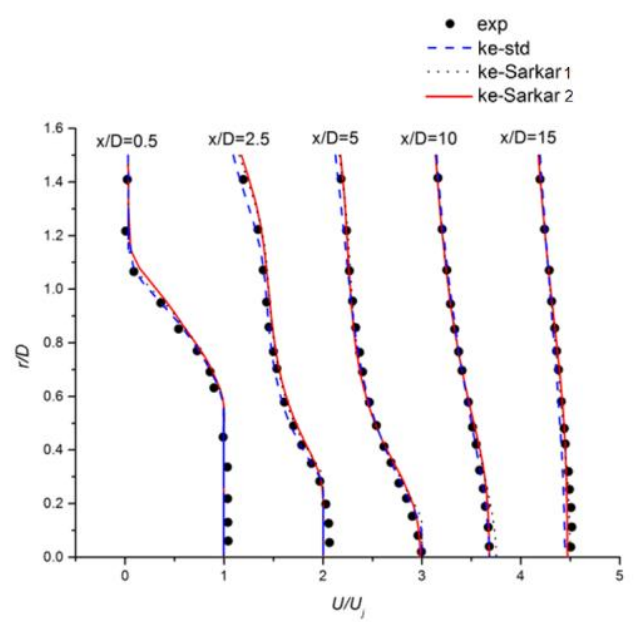

(b)

Fig. 8. Axial velocity distributions for the SMC006 case at the chevron valley plane. The experimental data are from the reference by Bridges and Brown (2004).

For the SMC006 case with chevron, the corresponding results are plotted in Fig. 13. The peak value of the total sound pressure level for SMC006 case is located at approximately $45^{\circ}$. The difference between the maximum sound pressure level and the minimum sound pressure level is about $7 \mathrm{~dB}$. Since there is no prediction point set at the location of $45^{\circ}$, the standard k- $\varepsilon$ model predicts the peak of the sound pressure level at around $40^{\circ}$ azimuth, and the peak value of the other two modified models is at approximately $50^{\circ}$ azimuth angle. As the turbulent kinetic energy in the SMC006 case is lower than in the SMC000 case towards the end of the potential core, the differences between the predictions from the three models is less than in the SMC000 case. The calculated results of the standard $\mathrm{k}-\varepsilon$ model are higher than those of the experimental data, and the maximum difference is about $4.1 \mathrm{~dB}$. The results of the present Sarkar2 model are in good agreement with experimental data, and the maximum difference is around $2.2 \mathrm{~dB}$. It can be concluded that the SNGR method can effectively predict the farfield noise of the chevron nozzle flow. Further, with improving the prediction accuracy of the turbulence and velocity flow fields, the predictions of the acoustic noise can be improved significantly.

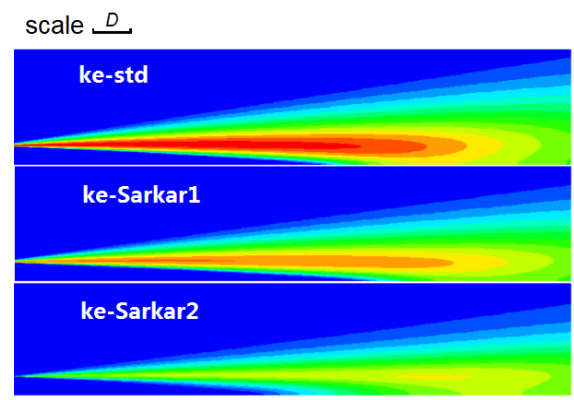

$200 \quad 400 \quad 600 \quad \frac{1}{200} 100012001400160018002000220024002600 \mathrm{~m}^{2} / \mathrm{s}^{2}$

(a)

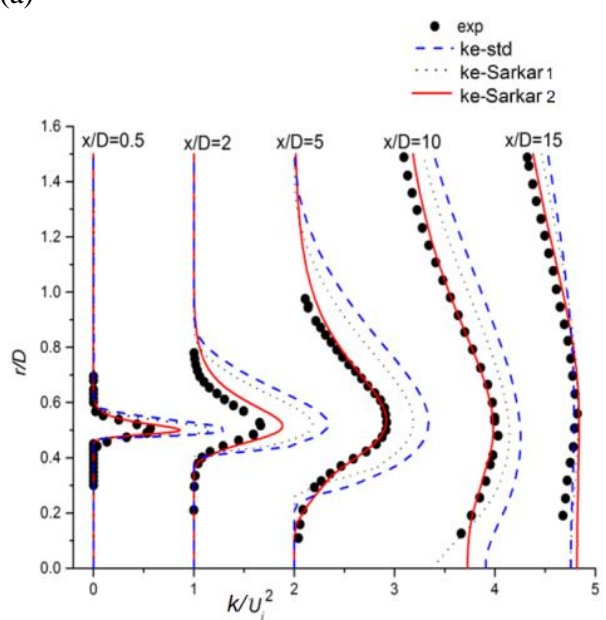

(b)

Fig. 9. Turbulent kinetic energy results for the SMC000 case. The experimental data are from the reference by Bridges and Brown (2004).

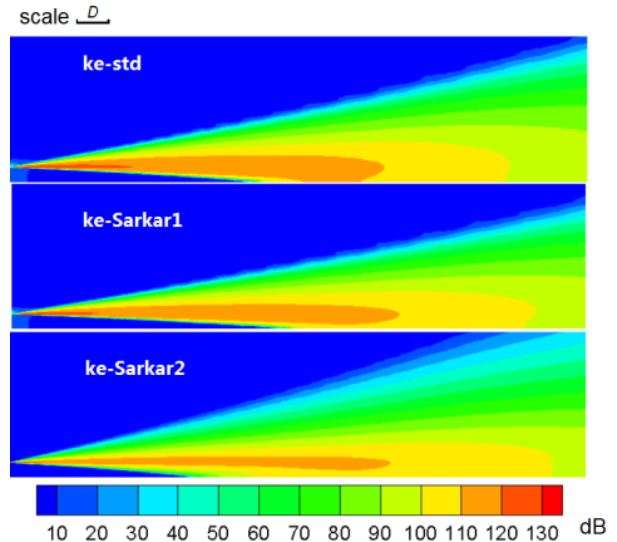

Fig. 10. Acoustic power levels for the SMC000 case at the central plane. 


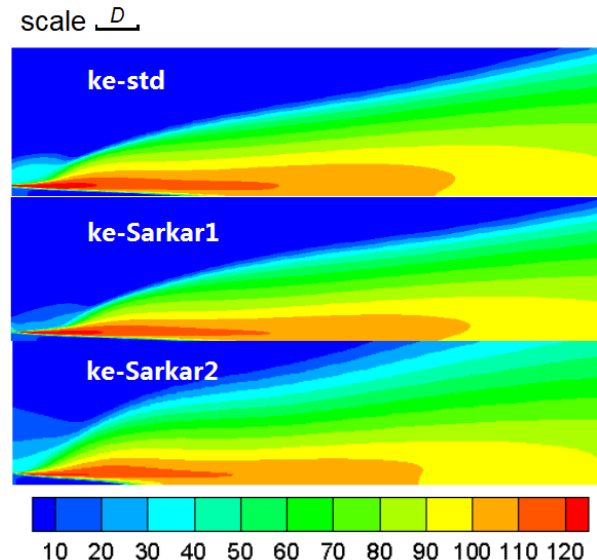

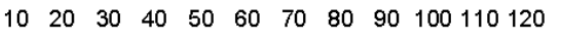

(a) scale $D$

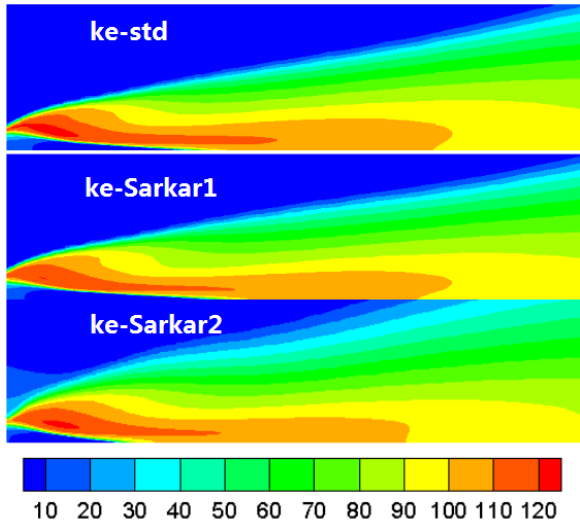

(b)

Fig. 11. Acoustic power levels for the SMC006 case at the chevron tip (a) and valley (b) central planes.

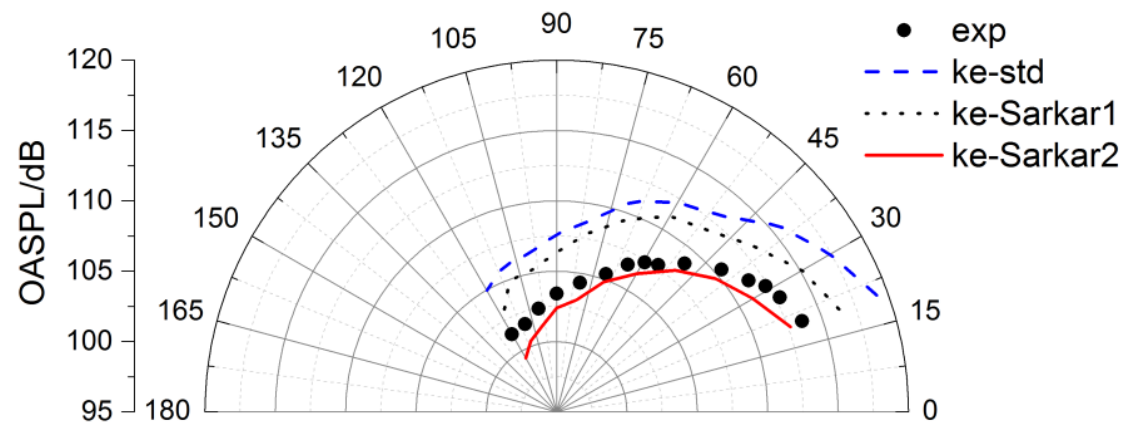

Fig. 12. Comparisons of the far-field acoustic noise for the SMC000 case. The experimental data are from the reference by Bridges and Brown (2004).

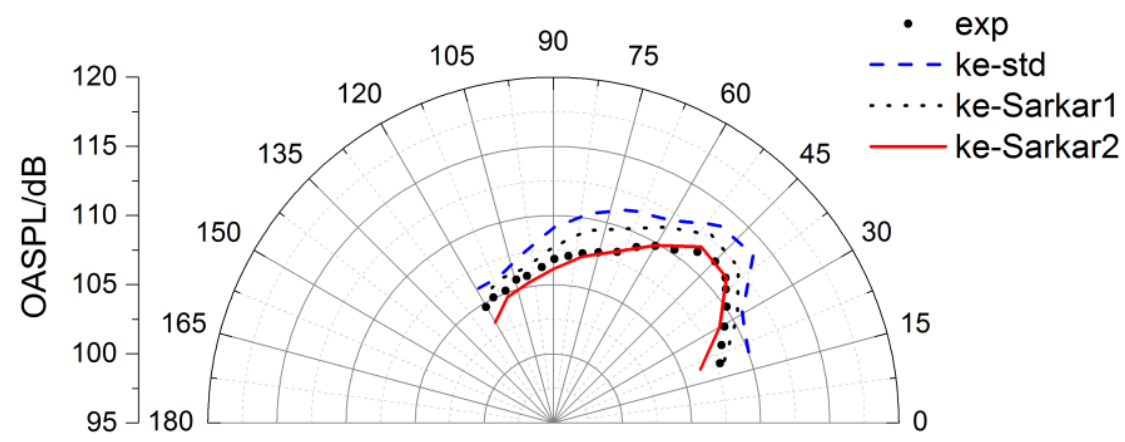

Fig. 13. Comparisons of the far-field acoustic noise for the SMC006 case. The experimental data are from the reference by Bridges and Brown (2004).

To analyze the effectivity of chevrons to reduce jet noise, the definition $\triangle$ OASPL $=$ OASPLSMC000 OASPLSMc006 is introduced. The variation along the azimuth is shown in Fig. 14. It can be seen that the prediction difference between the three turbulence models is significant $(>3 \mathrm{~dB})$. The present Sarkar2 model with three modification terms predicts the acoustic noise generation well compared with experiments $( \pm 1 \mathrm{~dB})$. As the results of numerical calculation are in good agreement with the experimental results, the present method based on the SNGR approach is found suitable for the study of noise reduction.

\section{Conclusions}

In the present study, the effects of compressibility modifications on the calculation of high subsonic jet flows are investigated based on the standard k$\varepsilon$ model. Further, the effect of the modifications on the acoustic noise prediction and reduction is 
analyzed based on the SNGR method. The following conclusions can be drawn:

(1) The compressibility modification has significant impact on the accuracy of jet flow and acoustic noise predictions. The present Sarkar2 turbulence model with three compressibility modification terms predicts accurately the jet flow and the farfield noise in the case of the chevron nozzles.

(2) In the RANS framework, the prediction accuracy of the acoustic noise relies on the accurate prediction of the turbulence and velocity flow field. The flow around the chevron nozzle is complex. The flow mixing is strongly enhanced by the geometry. The flow characteristics are predicted in better agreement with the experimental data than those in the circular nozzle case.

(3) The SNGR method can be used to predict the far-field acoustic noise of high subsonic jet flows consistently. It can be used even for sophisticated applications, such as chevrons. To study the effects of geometric modifications, e.g. design of chevrons for acoustic noise reduction, the SNGR method is a good candidate with high accuracy and low computational cost.

(4) The introduction of compressibility modifications into RANS turbulence closures and the SNGR method can benefit the study of acoustic noise generation in engineering applications.

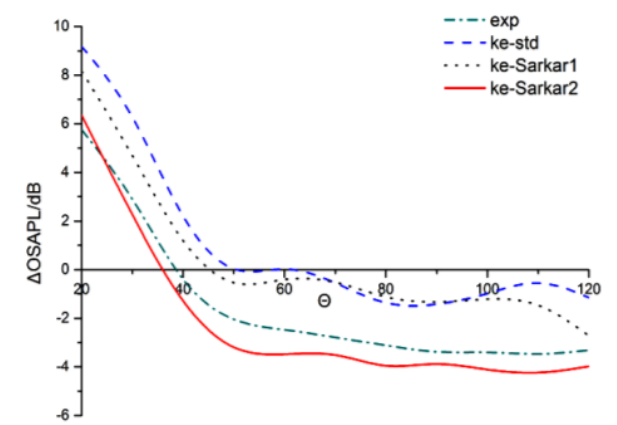

Fig. 14. Difference of the OASPL for the two cases of SMC000 and SMC006. The experimental data are from the reference by Bridges and Brown (2004).

\section{REFERENCES}

ANSYS Fluent, Theory Guide, Release 13.0, 2010.

Bahman-Jahromi, I., K. Ghorbanian and M. Ebrahimi (2019). Experimental Investigation on Acoustic Wave Generation due to Supersonic Hot Jet Impingement on an Inclined Flat Plate. Journal of Applied Fluid
Mechanics 12, 1063-1072.

Bailly, C., P. Lafon and S. Candel (1995). A stochastic approach to compute noise generation and radiation of free turbulent flows. AIAA paper, AIAA-95-029.

Bailly, C., S. Candel and P. Lafon (1997). Subsonic and Supersonic Jet Noise Predictions from Statistical Source Models. AIAA Journal 35, 1688-1696.

Balsa, T. F., P. R. Gliebe, R. A. Kantola, R. Mani and E. J. Stringas (1978). High Velocity Jet Noise Source Location and Reduction. Task 2. Theoretical Developments and Basic Experiments. Prepared by C.T. Savell et al. General Electric Company, Advanced Engineering and Technology Program Department.

Bastos, L. P., C. J. Deschamps and A. R. da Silva (2017). Experimental investigation of the far-field noise due to jet-surface interaction combined with a chevron nozzle. Applied Acoustics 127, 240-249.

Bechara, W., C. Bailly, P. Lafon and S. M. Candel (1994). Stochastic approach to noise modeling for free turbulent flows. AIAA Journal 32, 455-463.

Bose, T. (2013). Aerodynamic Noise: An Introduction for Physicists and Engineers. Springer-Verlag New York.

Bres, G. A. and S. K. Lele (2019). Modelling of jet noise: a perspective from large-eddy simulations. Philosophical Transactions of The Royal Society A 377, 20190081.

Bridges, J. and C. Brown (2004). Parametric Testing of Chevrons on Single Flow Hot Jets. AIAA paper, AIAA - 2004 - 2824.

Bridges, J. and M. Wernet (2011). The NASA Subsonic Jet Particle Image Velocimetry (PIV) Dataset. Technical report, NASA/TM 2011-216807.

Chassaing, P., R. A. Antonia, F. Anselmet, L. Joly and S. Sarkar (2002). Variable density fluid turbulence. Springer.

Demetriades, A. (1970). Turbulence measurements in supersonic two dimensional wake. Physics of Fluids 13, $1672-1678$.

Dhamankar, N. S., G. A. Blaisdell and A. S. Lyrintzis(2016). Analysis of Turbulent Jet Flow and Associated Noise with Round and Chevron Nozzles using Large Eddy Simulation. AIAA paper, AIAA 2016-3045.

Dussauge, J. P., P. Dupont and J. F. Debieve (2006). Unsteadiness in shock wave boundary layer interactions with separation. Aerospace Science and Technology 10, 8591.

Engel, R. C., C. R. Silva and C. J. Deschamps 
(2014). Application of RANS-based method to predict acoustic noise of chevron nozzles. Applied Acoustics 79, 153-163.

Erlebacher, G., M. Y. Hussaini, H. O. Kreiss and S. Sarkar(1990). The analysis and simulation of compressible turbulence. Theoretical and Computational Fluid Dynamics 2, 73-95.

Freund, J. B. (2001). Noise sources in a lowReynolds-number turbulent jet at Mach 0.9. Journal of Fluid Mechanics 438, 277-305.

Gatski, T. B. and J. Bonnet (2009). Compressibility, turbulence and high speed flow. Elsevier.

Han, X. S., T. H. Ye, M. M. Zhu and Y. L. Chen (2008). A new compressibility modification $\mathrm{k}-\varepsilon$ turbulence model with shock unsteadiness effect. Chinese Science Bulletin 53, 3798-3807.

Han, X. S., T. H. Ye and Y. L. Chen (2015). Effects of self-throttling on combustion enhancement in supersonic flow with transverse injection. International Journal of Hydrogen Energy 40, 8193-8205.

Henderson, B. S. (2010). Fifty Years of Fluidic Injection for Jet Noise Reduction. International Journal of Aeroacoustics 9, 91-122.

Kovasznay, L. S. G. (1953). Turbulence in supersonic flow. Journal of the Aeronautical Sciences 20, 657-674.

Krishnamurty, V. S. and W. Shyy (1997). Study of compressibility modification to the k-E turbulence model. Physics of Fluids 9, 27692788 .

Lele, S. K. (1994). Compressibility effects on turbulence. Annual Review of Fluid Mechanics, 211-254

Lighthill, M. J. (1954). On Sound Generated Aerodynamically. II. Turbulence as a Source of Sound. Proceedings of the Royal Society of London 222, 1-32.

Lilley, G. M. (1993). The radiated noise from isotropic turbulence revisited. NASA Contract Report, 93-75, NASA Langley Research Center, Hampton, VA.

Maizi, M., R. Dizene and M. C. Mihoubi (2017). Reducing Noise Generated from a Wind Turbine Blade by Pitch Angle Control using CFD and Acoustic Analogy. Journal of Applied Fluid Mechanics 10, 1201-1209.

Mesbah, M., J. Meyers, M. Baelmans and W. Desmet (2004). Assessment of different parameters used in the SNGR method. Proceedings of the International Conference on Noise and Vibration Engineering, Sept. 20-22 2004, Leuven, Belgium.

Mihaescu, M., B. Semlitsch, L. Fuchs and E. J. Gutmark (2012). Assessment of Turbulence
Models for Predicting Coaxial Jets relevant to Turbofan Engines. Conference on Modelling Fluid Flow (CMFF 12), 716-723, Budapest, Hungary.

Proudman, I. (1952). The Generation of Noise by Isotropic Turbulence. Proceedings of the Royal Society A 214, 119-132.

Raizada, N. and P. Morris (2006). Prediction of Noise from High Speed Subsonic Jets Using an Acoustic Analogy. AIAA paper, AIAA2006-2596.

Rosa, V. H., C. J. Deschamps, J. P. Salazar and da C. R. I. Silva (2013). Comparison of RANS-based methods for the prediction of noise emitted by subsonic turbulent jets. AIAA paper, AIAA 2013-2276.

Sarkar, S. (1992). The pressure-dilatation correlation in compressible flows. Physics of Fluids A 4, 2674 - 2682.

Sarkar, S., G. Erlebacher and M. Y. Hussaini (1991). Direct simulation of compressible turbulence in a shear flow. Theoretical and Computational Fluid Dynamics 2, 291-305.

Semlitsch, B. and M. Mihaescu (2018). Fluidic Injection Scenarios for Shock Pattern Manipulation in Exhausts. AIAA Journal 56 (12), 4640-4644.

Semlitsch, B., D. Cuppoletti, E. J. Gutmark and M. Mihaescu (2019). Transforming the Shock Pattern of Supersonic Jets Using Fluidic Injection. AIAA Journal 57 (5), 1851-1861.

Sinha, K., K. Mahesh and G. V. Candler (2003). Modeling shock unsteadiness in shock/turbulence interaction. Physics of Fluids 15, 2290- 2297.

Slessor, M. D., M. Zhuang and P. E. Dimotakis, (2000). Turbulent shear-layer mixing; growth-rate compressibility scaling. Journal of Fluid Mechanics 414, 35-45.

Tam, C. K. W. and L. Auriault (1999). Jet Mixing Noise from Fine-Scale Turbulence. AIAA Journal 37, 145-153.

Tam, C. K. W., K. Viswanathan, K. K. Ahuja and J. Panda (2008). The sources of jet noise: experimental evidence. Journal of Fluid Mechanics 615, 253-292.

Uzun, A. and M. Y. Hussaini (2012). Simulation of Noise Generation in the Near Nozzle Region of a Chevron Nozzle Jet. AIAA Journal 47, 1793-1810.

Venkatesh, B. J. and R. H. Self (2015). Parametric Study of Jet Nozzles Using a RANS-Based Jet Noise Prediction Tool. AIAA paper, AIAA 2015-2372.

Xia, H., P. G. Tucker and S. Eastwood (2009). Large-eddy simulations of chevron jet flows with noise predictions. International Journal 
Y. Jin et al. / JAFM, Vol. 14, No. 3, pp. 793-804, 2021.

of Heat and Fluid Flow 30, 1067-1079.

Zeman, O. (1991). On the decay of compressible isotropic turbulence. Physics of Fluids A 3, 951-955.

Zhao, K., S. Alimohammadi, P. N. Okolo, J. Kennedy and G. J. Bennett (2018). Aerodynamic noise reduction using dual-jet planar air curtains. Journal of Sound and
Vibration 432, 192-212.

Zuo, Z., Q. Huang and S. Liu (2019). An Analysis on the Flow Field Structures and the Aerodynamic Noise of Airfoils with Serrated Trailing Edges Based on Embedded Large Eddy Flow Simulations. Journal of Applied Fluid Mechanics 12, 327-339. 